\title{
Investigating the feasibility of exergame on sleep and emotion among university students
}

\begin{abstract}
Sleep deprivation and emotional problems such as stress, anxiety, and depression commonly occur in university students. Exercise is beneficial to ameliorate those problems; however, university students are not serious to take up physical activity. Commercially available exergame such as Xbox ${ }^{\circledR} 360$ Kinect is one of the alternatives. This study aims at investigating the feasibility and the potential efficacy of using Xbox 360 Kinect game among health care undergraduate students. A pilot two-armed parallel randomized controlled trial was implemented. A total of 36 undergraduate students was recruited and randomly allocated into the intervention group (playing Xbox 360 Kinect) or the control group (continue with normal daily routine). The intervention group received 30 minutes of Xbox Kinect activity, three times per week for 6 weeks. Information on psychology (Depression, Anxiety, and Stress Scale-21) and sleep (Functional Outcome Sleep Questionnaire-30) status was collected at pre- and postexperiment. The researcher-developed feasibility questionnaire was given to the participants in the intervention group at post-experiment. Repeated-measures analysis of variance was used to investigate within-between group comparison, and significance value was set at $p \leq 0.05$. The analysis found potential improvement on sleep $(\mathrm{p}=0.039)$ and psychological health $(p=0.002-0.067)$. The intervention protocol is feasible and highly accepted by the participants. The required optimum amount of dosage, sample size, and the use of outcome measures are suggested from the findings. This pilot and feasibility study supports the use of Xbox 360 Kinect games in practice and to be implemented for future research.
\end{abstract}

Keyword: Exercise; Leisure; Pilot study; Randomized controlled trial; Xbox Kinect 